\title{
DISK ROTATION CURVES IN TRIAXIAL POTENTIALS
}

\author{
Ortwin E. GERHARD ${ }^{1}$ and Mario VIETRI ${ }^{2}$ \\ ${ }^{1}$ Max-Planck-Institut für Astrophysik, Garching, West Germany \\ ${ }^{2}$ Osservatorio Astrofisico, Firenze, Italy
}

ABSTRACT. The apparent rotation curve of cold gas in a triaxial potential often differs from that in an axisymmetric system in characteristic ways. The data argue for triaxial bulges in our Galaxy and several others.

Gas rotation on elongated closed orbits. A significant fraction of elliptical galaxies contain neutral or ionized gas (Knapp and Sadler, this volume). In the absence of shocks, this gas can be treated as a collisionless fluid orbiting on stable, closed, non-self-intersecting orbits (Sanders \& Huntley 1976). Here we consider motion in the equatorial plane of a non-rotating triaxial galaxy. Then the relevant closed orbits are the short axis loops, which are elongated along the intermediate axis of the potential. When viewed down its major axis (the potential's intermediate axis), the tangent point velocity of such an orbit is higher than that of the circular orbit at the same radius in a corresponding axisymmetric potential (Lake \& Norman 1982), while the reverse effect is seen when the orbit is viewed down its short axis. Thus in a triaxial potential, the apparent rotation curve is determined by the run with radius of $M / r$ and orbital ellipticity (as well as geometry). E.g. in a de Vaucouleurs-type profile or in the density profiles of galactic bulges the closed orbits become rapidly round outside a turnover radius in the density distribution, introducing characteristic features in the rotation curve. We now describe how this applies to our own Galaxy (Gerhard \& Vietri 1986; hereafter GV).

Model of the Galaxy. We use a triaxial bulge with $\rho(a) \propto a^{-1.8}$ inside $800 p c$ as determined by infrared observations, and $\rho(a) \propto a^{-3.5}$ for $a>800 p c$ as is consistent with these data and the local density of spheroid stars. We also include an exponential disk with scale-length $3.5 k p c$ and local surface density $64 M_{\odot} / p c^{2}$ (see GV). Fig. 1 shows the apparent rotation curves from loop orbits in three bulge models, spherical, axisymmetric, and non-axisymmetric, with the same peak velocity, as seen by an observer on the intermediate axis of the potential. Also shown is the Galactic rotation curve from $\mathrm{HI}$ and $\mathrm{CO}$ terminal velocities. It is clear that the observed galactic rotation curve is consistent with the $2.4 \mu \mathrm{m}$ bulge density profile and the local density of spheroid stars only if the bulge is non-axisymmetric. In this model, the orbital ellipticity is $\sim 20 \%$ inside $\sim 600 p c$ and then decreases rapidly with $r$. Thus the peak in the rotation curve is not at the knee in the density profile as in the axisymmetric models, but closer to the centre $(r=500 \mathrm{pc})$. The Sun need not be aligned with the bulge intermediate 
axis exactly. Fig. 2 shows the apparent tangent velocity curves for observers at various angles $\phi$ wrt this axis. A range $-20^{\circ} \lesssim \phi \lesssim 20^{\circ}$ seems acceptable for the solar position (with scaled $M / L$ ). Fig. 2 also shows that from a viewing direction along the potential's major axis the rotation curve rises unusually slowly.

Bulges and elliptical galaxies. The characteristic morphology of a peak and subsequent near-Keplerian fall-off in the rotation curve is also shown by NGC 3200 and UGC 2885. The two galaxies NGC 2708 and NGC 3054 have such slowly rising rotation curves, that the fits by an axisymmetric model based on CCD photometry appear to require negative $M / L$ ratios for their bulges (Kent 1986). With triaxial bulges this is expected for lines-of-sight in the plane containing the major and minor axes of the potential $\left(\phi=90^{\circ}\right.$ in Fig. 2). Thus triaxiality of the bulge is probably important in modelling the rotation curves of at least a fraction of spiral galaxies, and may significantly affect the derived mass-to-light ratios.

In elliptical galaxies with a de Vaucouleurs profile the transition from elongated to nearly circular orbits occurs in the range between a fraction of an $r_{e}$ and two or three $r_{e}$. The closed orbits in the inner part of the system are significantly more elongated than in the Galactic model of the same ellipticity; thus if gas is found on these orbits, the expected effects are significantly larger. Again the characteristic morphology indicating triaxiality is either a peak-fall-off structure or a very slow rise of the rotation curve, both inconsistent with constant $M / L$ inside $\sim 2 r_{e}$.
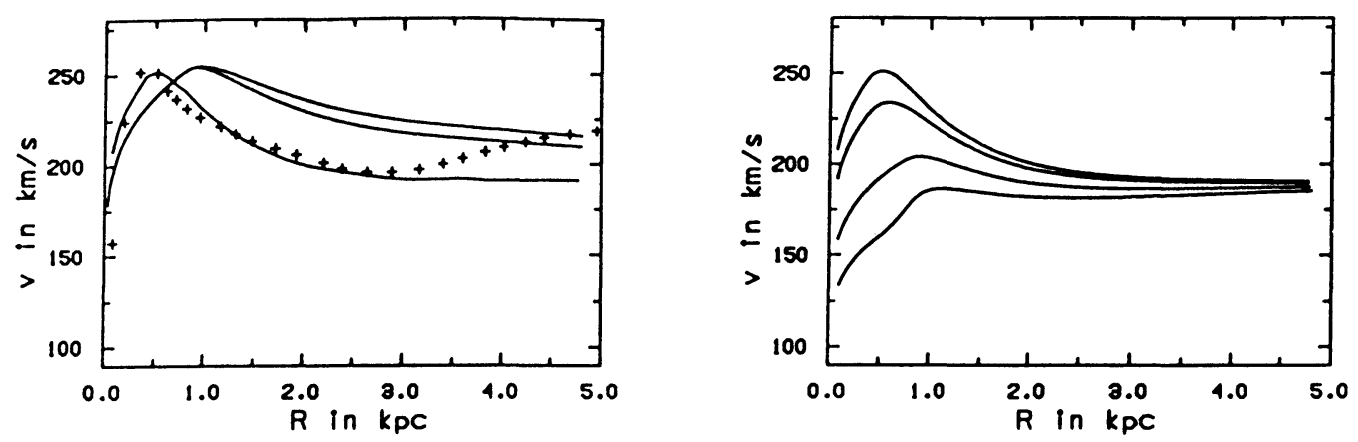

Fig. 1 (left): Apparent rotation curves along the intermediate axis for three galactic bulge models plus galactic disk, and compared with observations. Crosses are taken from a smooth curve drawn through the data points (Clemens 1985). Curves from top to bottom at large radii: Spherical, oblate $(1: 1: 0.4)$, and prolate-barred $(1: 0.6: 0.6)$ bulge.

Fig.2 (right): Tangent rotation curves in a prolate bulge (1:0.6:0.6) plus disk model, for observers at $\infty$ and with different position angles $\phi$ relative to the bulge's intermediate axis. Curves are (from top) for $\phi=0^{\circ}, 20^{\circ}, 45^{\circ}, 90^{\circ}$.

\section{REFERENCES}

Clemens, D.P., 1985. Astrophys. J. 295, 422.

Gerhard, O.E., Vietri, M., 1986. Mon. Not. R. astr. Soc., 223, 377.

Kent, S.M., 1986. Astron. J. 91, 1301.

Lake, G., Norman, C., 1982. in The Galactic Center, A.I.P. Conference no. 83, ed. G.R. Riegler, R.D. Blandford, p.189.

Sanders, R.H., Huntley, J.M., 1976. Astrophys. J. 209, 53. 